\title{
Discrete 2D Fourier Transform in Polar Coordinates
}

\author{
Xueyang Yao \\ Department of Mechanical Engineering \\ University of Ottawa \\ Ottawa, Canada \\ xyao011@uottawa.ca
}

\author{
Natalie Baddour \\ Department of Mechanical Engineering \\ University of Ottawa \\ Ottawa, Canada \\ nbaddour@uottawa.ca
}

\begin{abstract}
The discrete Fourier transform in Cartesian coordinates has proved to be invaluable in many disciplines. However, less theory has been developed for functions that are best described in polar coordinates. In this paper, a discrete $2 \mathrm{D}$-Fourier transform in polar coordinates is proposed and tested by numerical simulations with respect to accuracy and precision. Guidelines for choosing sample size are developed.
\end{abstract}

Keywords-2D Fourier Transform, discrete, polar coordinates

\section{INTRODUCTION}

The Fourier transform is a powerful analytical tool and has proved to be invaluable in many disciplines such as physics, mathematics and engineering. The development of the Fast Fourier Transform (FFT) algorithm [1], which computes the discrete Fourier transform with a fast algorithm, established the Fourier transform as a practical tool in diverse areas, most notably signal and image processing.

In two dimensions, the FFT can still be used to compute the discrete Fourier transform in Cartesian coordinates. However, in many applications such as photoacoustics [2] and tomography ([3], [4], [5]), it is often necessary to compute the Fourier transform in polar coordinates. Moreover, for functions that are naturally described in polar coordinates, a discrete version of the 2D Fourier transform in polar coordinates is needed.

There have been some attempts to calculate the Fourier transform in polar coordinates. Nonequally spaced FFTs (NUFFT, see [6], [7], [8], [9]) is one of the methods that can be implemented to compute the FFT in polar coordinates. However, due to local interpolations, the NUFFT can be prohibitively slow for large input sizes and is not easily inverted. Averbuch et al. [10] proposed a new discrete polar Fourier transform, where the original function was sampled on a near-polar grid called a pseudo-polar grid followed by 1D equispaced FFT and 1D interpolations. Based on Averbuch's work, this new method was more accurate than the NUFFT method.

It should be noted that prior work has focused on numerically approximating the continuous transform. This stands in contrast to approaches that have been taken with the continuous/discrete Fourier transform in two aspects: 1. both functions in the space and frequency domain of the DFT are in the same coordinates. 2. The DFT is defined as a transform in its own right, which means the existence of the DFT is valid even if without the existence of the continuous Fourier transform. In this paper, a new discrete 2D-Fourier transform in polar coordinates is proposed and tested by numerical simulations with respect to accuracy and precision.

\section{DEFNITION OF THE DISCRETE 2D FOURIER TRANSFORM IN POLAR COORDINATES}

\section{A. Kernel of the discrete 2D Fourier transform in polar} coordinates

To propose and work with a $2 \mathrm{D}$ polar DFT, the following kernels are proposed

$$
\begin{aligned}
& E^{-}(q l ; p k)=\frac{2}{N_{2}} \sum_{n=-M}^{M} \frac{J_{n}\left(\frac{j_{n k} j_{n l}}{j_{n N_{1}}}\right)}{j_{n N_{1}} J_{n+1}^{2}\left(j_{n k}\right)} i^{-n} e^{-i n \frac{2 \pi p}{N_{2}}} e^{+i n \frac{2 \pi q}{N_{2}}} \\
& E^{+}(q l ; p k)=\frac{2}{N_{2}} \sum_{n=-M}^{M} \frac{J_{n}\left(\frac{j_{n l} j_{n k}}{j_{n N_{1}}}\right)}{j_{n N_{1}} J_{n+1}^{2}\left(j_{n l}\right)} i^{n} e^{+i \frac{2 \pi n p}{N_{2}}} e^{-i \frac{2 \pi n q}{N_{2}}}
\end{aligned}
$$

where $p, k, q, l, n, N_{1}$ and $N_{2}$ are integers such that $-M \leq n \leq M$, where $2 M+1=N_{2}, 1 \leq l, k, \leq N_{1}-1$ and $-M \leq p, q \leq M . J_{n}()$ is the Bessel function of $n t h$ order and $j_{n k}$ is the kth zero of the Bessel function of order $n$. The integers $N_{1}$ and $N_{2}$ represent the size of the spaces in which we work, with $N_{2}$ representing the dimension in the angular direction and $N_{1}$ represents the dimension in the 
radial direction. Since $N_{2}=2 M+1, N_{2}$ must be an odd integer.

\section{B. Definition of the discrete transform}

The 2D-Discrete Fourier Transform in polar coordinates is defined as the discrete transform that transforms the matrix (or double-subscripted series $f_{p k}$ to the matrix (double-subscripted series) to the matrix $F_{q l}$ according to

$$
\begin{aligned}
& F_{q l}=\mathbb{F}\left(f_{p k}\right) \\
& F_{q l}=\sum_{k=1}^{N_{1}-1} \sum_{p=-M}^{M} f_{p k} E^{-}(q l ; p k) \\
& =\sum_{k=1}^{N_{1}-1} \sum_{p=-M}^{M} f_{p k} \frac{2}{N_{2}} \sum_{n=-M}^{M} \frac{J_{n}\left(\frac{j_{n k} j_{n l}}{j_{n N_{1}}}\right) i^{-n}}{j_{n N_{1}} J_{n+1}^{2}\left(j_{n k}\right)} e^{-i n \frac{2 \pi p}{N_{2}}} e^{+i n \frac{2 \pi q}{N_{2}}}
\end{aligned}
$$

The notation for $E^{-}(q l ; p k)$ and $E^{+}(q l ; p k)$ are the discrete kernels for the forward and inverse transform, respectively. The subscript (+ or -) indicates the sign on the exponent containing the $p$ variable; the $q$ variable exponent then takes the opposite sign. From a matrix point of view, both $f_{p k}$ and $F_{q l}$ are $N_{2} \times\left(N_{1}-1\right)$ sized matrices. The inverse transform is then given by

$$
\begin{aligned}
f_{p k} & =\mathbb{F}^{-1}\left(F_{q l}\right) \\
f_{p k} & =\sum_{l=1}^{N_{1}-1} \sum_{q=-M}^{M} F_{q l} E^{+}(q l ; p k) \\
= & \sum_{l=1}^{N_{1}-1} \sum_{q=-M}^{M} F_{q l} \frac{2}{N_{2}} \sum_{n=-M}^{M} \frac{J_{n}\left(\frac{j_{n l} j_{n k}}{j_{n N_{1}}}\right) i^{n}}{j_{n N_{1}} J_{n+1}^{2}\left(j_{n l}\right)} e^{+i \frac{2 \pi n p}{N_{2}}} e^{-i \frac{2 \pi n q}{N_{2}}}
\end{aligned}
$$

\section{Approximation to the continuous Fourier transform}

To approximate the continuous Fourier transform, the function needs to be sampled at some specific points. For a space limited function defined over continuous $(r, \theta)$ space where $0 \leq r \leq R$ and $0 \leq \theta \leq 2 \pi$ and the function is effectively zero elsewhere (that is the function can be made as close to zero as necessary, elsewhere in the plane), the sampling points are defined in the spatial domain as

$$
r_{p k}=\frac{j_{p k} R}{j_{p N_{1}}} \quad \theta_{p}=\frac{p 2 \pi}{N_{2}}
$$

And in the spatial frequency domain as

$$
\rho_{q l}=\frac{j_{q l}}{R} \quad \psi_{q}=\frac{q 2 \pi}{N_{2}}
$$

Equations (4) and (5) give the sampling grid in regular $(r, \theta)$ and frequency $(\rho, \psi)$ space. Clearly, the density of the sampling points depends on the numbers of points chosen, that is on $N_{1}$ and $N_{2}$. Also clear is the fact that the grid is not equi-spaced in the radial variable. It can be shown that the approximate relationship between sampled values of the continuous function $f(r, \theta)$ and sampled values of its continuous forward 2D transform $F(\rho, \psi)$ is given by

$F\left(\frac{j_{q l}}{R}, \frac{2 \pi q}{N_{2}}\right) \approx 2 \pi R^{2} \sum_{k=1}^{N_{1}-1} \sum_{p=-M}^{M} f\left(\frac{j_{p k} R}{j_{p N_{1}}}, \frac{2 \pi p}{N_{2}}\right) E^{-}(q l ; p k)$

Similarly, the inverse transform relationship is given as

$$
f\left(\frac{j_{p k} R}{j_{p N_{1}}}, \frac{2 \pi p}{N_{2}}\right) \approx \frac{1}{2 \pi R^{2}} \sum_{l=1}^{N_{1}-1} \sum_{q=-M}^{M} F\left(\frac{j_{q l}}{R}, \frac{2 \pi q}{N_{2}}\right) E^{+}(q l ; p k)
$$

Consider the case of a bandlimited function, such that the function is defined over continuous $(\rho, \psi)$ space where $0 \leq \rho \leq W_{p}$ and $0 \leq \psi \leq 2 \pi$ and the function is effectively zero elsewhere (that is the function can be made as close to zero as necessary, elsewhere in the plane), the sampling points are defined as

$$
r_{p k}=\frac{j_{p k}}{W_{p}} \quad \theta_{p}=\frac{p 2 \pi}{N_{2}}
$$

and

$$
\rho_{q l}=\frac{j_{q l} W_{p}}{j_{q N_{1}}} \quad \psi_{q}=\frac{q 2 \pi}{N_{2}}
$$

The approximate relationship between sampled values of the continuous function and sampled values of its continuous forward 2D transform $F(\rho, \psi)$ can be shown to be given by

$$
F\left(\frac{j_{q l} W_{p}}{j_{q N_{1}}}, \frac{2 \pi q}{N_{2}}\right) \approx \frac{2 \pi}{W_{p}^{2}} \sum_{k=1}^{N_{1}-1} \sum_{p=-M}^{M} f\left(\frac{j_{p k}}{W_{p}}, \frac{2 \pi p}{N_{2}}\right) E^{-}(q l ; p k)
$$

Similary, the inverse transform is given as 


$$
f\left(\frac{j_{p k}}{W_{p}}, \frac{2 \pi p}{N_{2}}\right) \approx \frac{W_{p}^{2}}{2 \pi} \sum_{l=1}^{N_{1}-1} \sum_{q=-M}^{M} F\left(\frac{j_{q l} W_{p}}{j_{q N_{1}}}, \frac{2 \pi q}{N_{2}}\right) E^{+}(q l ; p k)
$$

\section{DisCRETE 2D FourieR TRANSFORM TEST AND RESUltS}

\section{A. Method for testing the Algorithm}

In this section, the ability of the 2D discrete Fourier transform is evaluated for its ability to estimate the continuous Fourier transform at the selected special sampling points in the spatial and frequency domains.

1) Accuracy

In order to test the accuracy of the 2D-DFT and 2D-IDFT to calculate the continuous counterpart, the dynamic error has been used. It is defined as [11]

$$
E(\mathrm{v})=20 \log _{10}\left[\frac{|C(\mathrm{v})-D(\mathrm{v})|}{\max |D(\mathrm{v})|}\right]
$$

where $C(\mathrm{v})$ is the continuous forward or inverse $2 \mathrm{D}$-Fourier transform and $D(\mathrm{v})$ is the values obtained from the discrete counterpart. The dynamic error is defined as the ratio of the absolute error to the maximum amplitude of the function, which is calculated on a log scale. Therefore, a large negative value represents an accurate discrete transform. The dynamic error is used instead of the percentage error in order to avoid division by zero.

\section{2) Precision}

The precision of the algorithm is another important evaluation criterion, which is tested by sequentially performing a pair of forward and inverse transforms and comparing the result to the original function. High precision indicates that the transform does not add much error by the calculations. An average of absolute error of each sample points between the original function and the calculated counterpart was used to measure the precision. It is given by

$$
\varepsilon=\frac{1}{\left(N_{1}-1\right) \cdot N_{2}} \sum_{n=1}^{\left(N_{1}-1\right) \cdot N_{2}}\left|f-f^{*}\right|
$$

where $f$ is the original function and $f^{*}$ is the calculated counterpart. An ideal precision would result in the absolute error being zero.

\section{B. Test Functions}

\section{1) Gaussian}

The first function chosen for evaluation is a circular symmetric function which is Gaussian in the radial direction. The function in the space domain is defined as

$$
f(r, \theta)=e^{-a^{2} r^{2}}
$$

where $a$ is some real constant. Since the function is circularly symmetric, the 2D-DFT is actually a zeroth-order Hankel Transform [12] and can be written as

$$
F(\rho, \psi)=\frac{\pi}{a^{2}} e^{\frac{-\rho^{2}}{4 a^{2}}}
$$

The graphs for the original function and its continuous 2DDFT (which is also a Gaussian) are shown with $a=1$ and shown in Figure 1.
Original Function

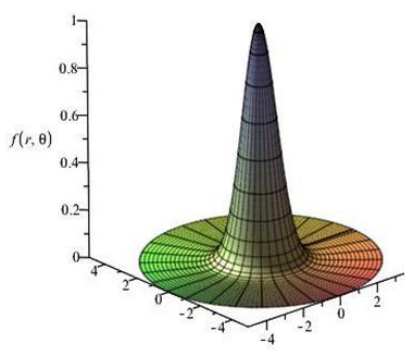

2D-Fourier Tranform

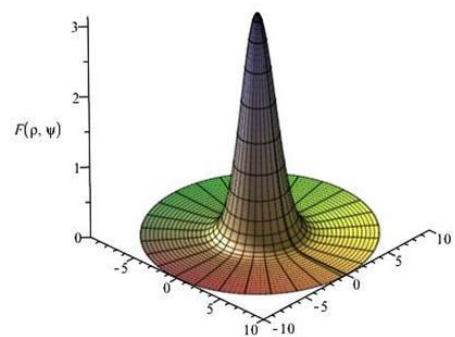

Figure 1. The original Gaussian function and its 2D-Fourier Transform
From Figure 1, the function is circular symmetric in the angular direction and fairly smooth in the radial direction. Moreover, the function can be considered as an effectively space limited function or an effectively band limited function. For the purposes of testing it, it shall be considered as a space limited function and (6) and (7) will be used to proceed with the forward and inverse transform in sequence.

To perform the transform, the following variables need to be chosen: $N_{2}, R$ and $N_{1}$. In the angular direction, since the function in the spatial domain is circularly symmetric, $N_{2}$ can be chosen to be small. Thus, $N_{2}=15$ is chosen.

In the radial direction, the effective space limit $R=40$ and effective band-limit $W_{p}=30$ are chosen, which gives $j_{0 N_{1}} \geq R \cdot W_{p}=1200$ [13]. Therefore, $N_{1}=383$ is chosen to satisfy this constraint.

\section{a) Forward Transform}

Test results for the forward transform are shown in Figure 2 and Figure 3. The error gets bigger at the center as expected. However, the maximum value of the error is $E_{\max }=-8.3842 \mathrm{~dB}$ and this occurs at the center. The average value of the error is $E_{\text {avg. }}=-63.8031 \mathrm{~dB}$. 


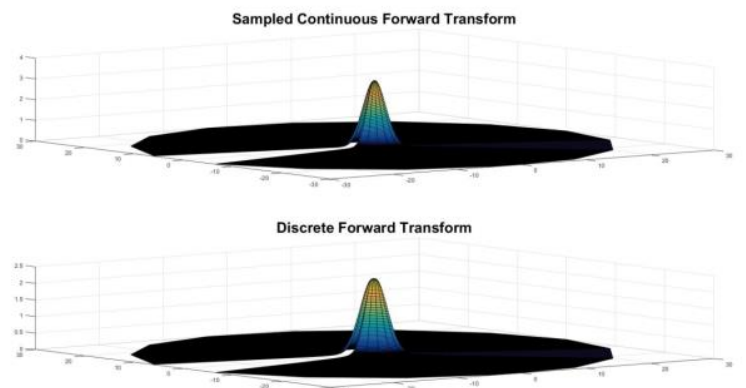

Figure 2. Sampled Continuous forward transform \&. Discrete forward transform of Gaussian Function with $\mathrm{R}=40, \mathrm{~N}_{2}=15, \mathrm{~N}_{1}=383$

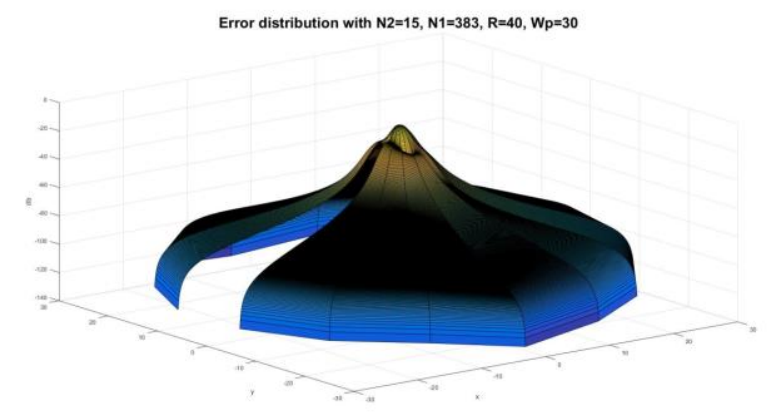

Figure 3. The error distribution of the forward transform of Gaussian Function with $\mathrm{R}=40, \mathrm{~N}_{2}=15, \mathrm{~N}_{1}=383$

\section{b) Inverse Transform}

Figure 4 and Figure 5 show the test results for the inverse transform. The maximum value of the error is $E_{\max }=-12.2602 \mathrm{~dB}$ and this occurs at the center. The average of the error is $E_{\text {avg. }}=-98.0316 \mathrm{~dB}$.

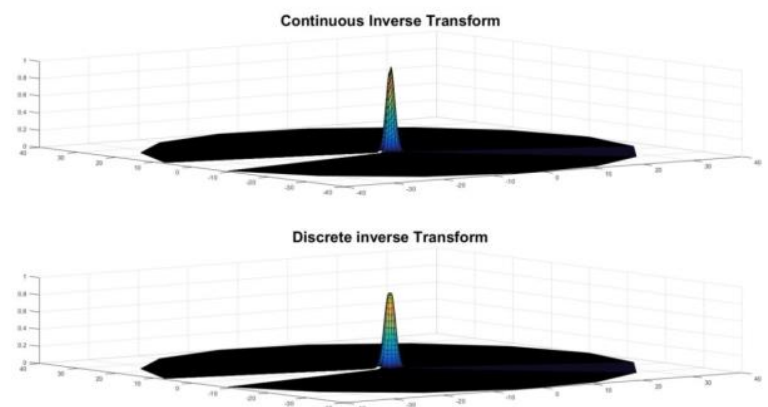

Figure 4. Sampled continuous inverse transform and discrete inverse transform of the Gaussian function with $R=40, N_{2}=15, N_{1}=383$

Performing sequential 2D-DFT and 2D-IDFT results in $\varepsilon=4.1656 \times e^{-17} \quad$ where $\varepsilon$ is calculated with (13). Therefore, performing the forward and inverse transforms does not add much error.

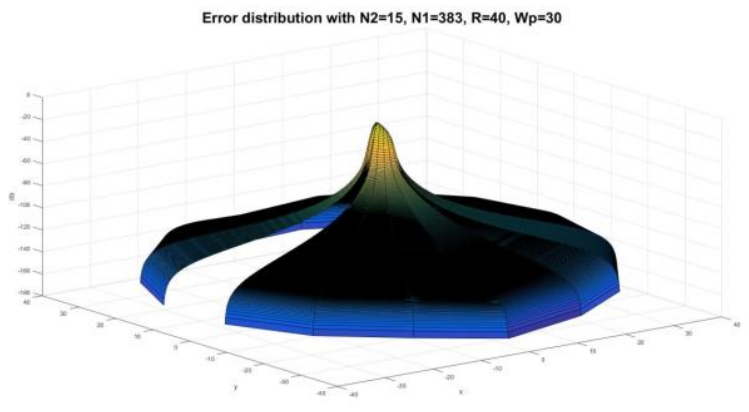

Figure 5. The error distribution of the inverse transform of Gaussian Function with $\mathrm{R}=40, \mathrm{~N}_{2}=15, \mathrm{~N}_{1}=383$

\section{2) Square Donut}

The second chosen function is a circularly symmetric function which is a square wave in the radial direction. The function is given by

$$
f(r, \theta)= \begin{cases}0, & r<5 \text { and } r>10 \\ 1, & 5 \leq r \leq 10\end{cases}
$$

The continuous 2D-FT can be written as:

$$
F(\rho, \psi)=\frac{2 \pi}{\rho}\left[10 J_{1}(10 \rho)-5 J_{1}(5 \rho)\right]
$$

where $J_{n}(x)$ is Bessel function of order $n$.

The graphs for the original function and its continuous 2DDFT (which is also a Gaussian) are plotted and shown in Figure 6.
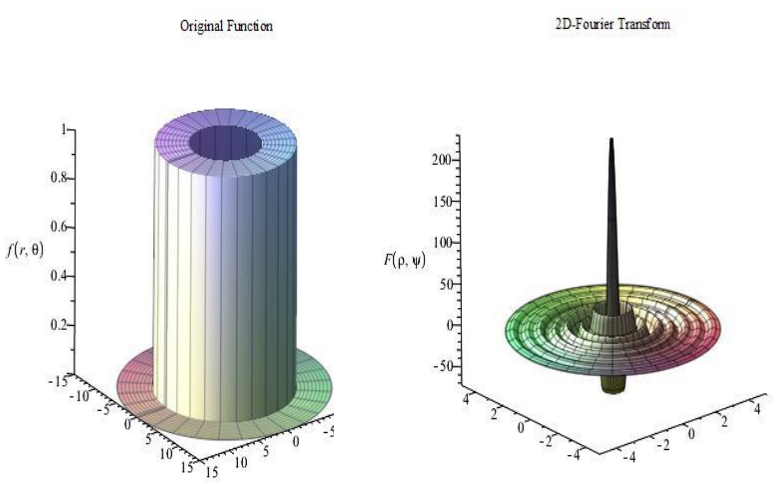

Figure 6. The original 'Square Donut' and its 2D-Fourier Transform

\section{a) Forward Transform}

Test results for the forward transform are shown in Figure 7 and Figure 8. Similar to the previous case, the error gets bigger at the center as expected. The maximum value of the error is $E_{\max }=-8.1664 d B$ and occurs at the center area. The average of the error is $E_{\text {avg. }}=-34.5471 \mathrm{~dB}$.

\section{b) Inverse Transform}

Figure 9 and Figure 10 show the test result for the inverse transform. The maximum value of the error is 
Error $_{\max }=1.5 \mathrm{~dB}$.The average of the error is Error $_{\text {average }}=-73 \mathrm{~dB}$.
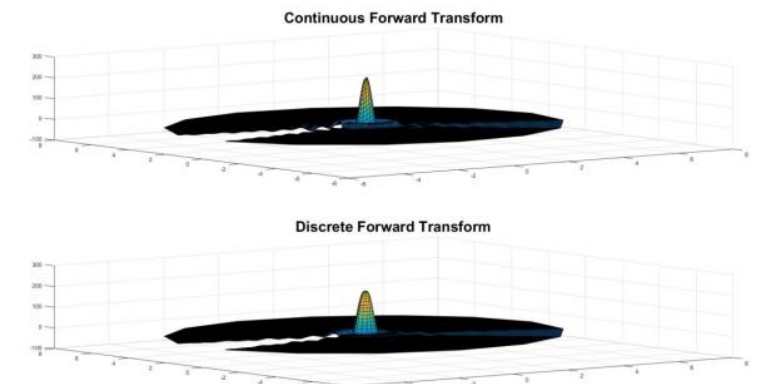

Figure 7. Sampled Continuous forward transform \&. Discrete forward transform of 'Square Donut' Function with $R=40, N_{2}=15, N_{1}=383$

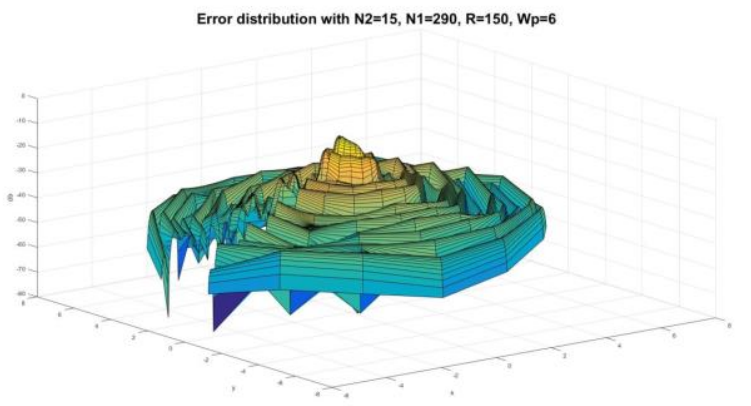

Figure 8. The error distribution of the forward transform of 'Square Donut' Function with $\mathrm{R}=40, \mathrm{~N}_{2}=15, \mathrm{~N}_{1}=383$
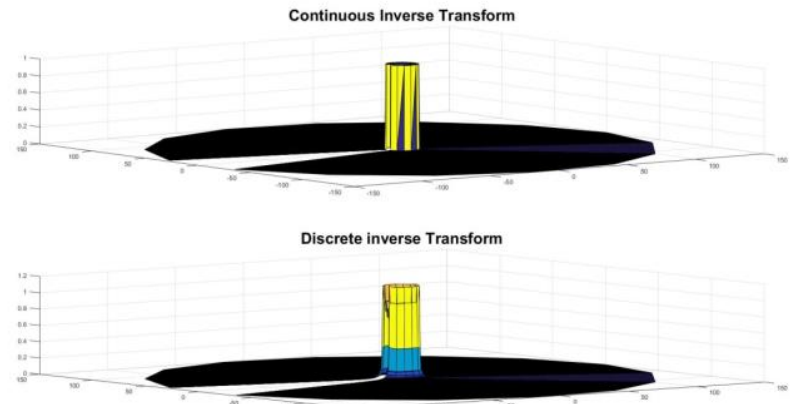

Figure 9. Sampled continuous inverse transform and discrete inverse transform of the 'Square Donut' function with $R=40, N_{2}=15, N_{1}=383$

Performing sequential 2D-DFT and 2D-IDFT results in $\varepsilon=6.7253 \times e^{-14} \quad$ where $\varepsilon$ is calculated with (13). Therefore, performing forward and inverse transform does not add much error.

\section{SUMMARY AND CONLUSION}

From the two test cases, both the forward and inverse transform showed good accuracy to approximate the continuous Fourier transform. Moreover, the good precision results indicated that the transform itself does not add much error.

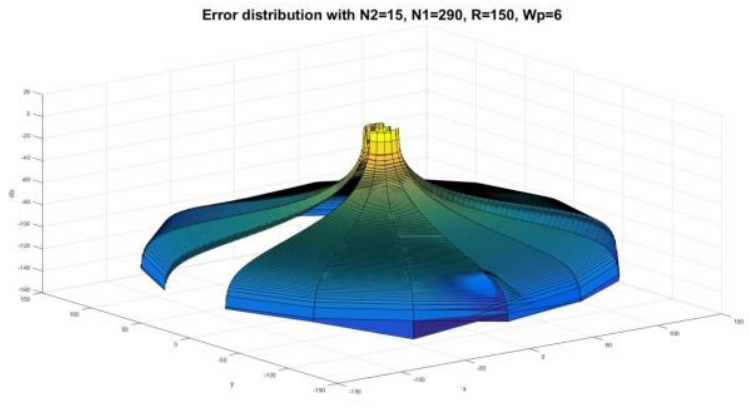

Figure 10. The error distribution of the inverse transform of 'Square Donut' Function with $\mathrm{R}=40, \mathrm{~N}_{2}=15, \mathrm{~N}_{1}=383$

\section{REFERENCES}

[1] J. W. Cooley and J. W. Tukey, "An Algorithm for the Machine Calculation of Complex Fourier Series," Math. Comput., vol. 19, no. 90, pp. 297-301, 1965.

[2] Y. Xu, D. Feng, and L. V. Wang, "Exact frequencydomain reconstruction for thermoacoustic tomography. I. Planar geometry," Med. Imaging IEEE Trans. On, vol. 21, no. 7, pp. 823-828, 2002.

[3] M. C. Scott et al., "Electron tomography at 2.4-ångström resolution," Nature, vol. 483, no. 7390, p. 444, Mar. 2012.

[4] B. P. Fahimian et al., "Radiation dose reduction in medical X-ray CT via Fourier-based iterative reconstruction," Med. Phys., vol. 40, no. 3, p. n/a-n/a, Mar. 2013.

[5] E. Lee et al., "Radiation dose reduction and image enhancement in biological imaging through equally-sloped tomography," J. Struct. Biol., vol. 164, no. 2, pp. 221-227, 2008.

[6] E. Suli and A. Ware, "A Spectral Method of Characteristics for Hyperbolic Problems," SIAM J. Numer. Anal., vol. 28, no. 2, pp. 423-445, 1991.

[7] A. Dutt and V. Rokhlin, "Fast Fourier Transforms for Nonequispaced Data," SIAM J. Sci. Comput., vol. 14, no. 6, pp. 1368-1393, Nov. 1993.

[8] C. Anderson and M. Dahleh, "Rapid Computation of the Discrete Fourier Transform," SIAM J. Sci. Comput., vol. 17, no. 4, pp. 913-919, Jul. 1996.

[9] A. F. Ware, "Fast Approximate Fourier Transforms for Irregularly Spaced Data," SIAM Rev., vol. 40, no. 4, p. 838, Dec. 1998.

[10] A. Averbuch, R. R. Coifman, D. L. Donoho, M. Elad, and M. Israeli, "Fast and accurate Polar Fourier transform," Appl. Comput. Harmon. Anal., vol. 21, no. 2, pp. 145-167, 2006.

[11] M. Guizar-Sicairos and J. C. Gutiérrez-Vega, "Computation of quasi-discrete Hankel transforms of integer order for propagating optical wave fields," JOSA A, vol. 21, no. 1, pp. 53-58, Jan. 2004.

[12] A. D. Poularikas, The Transforms and Applications Handbook. Boca Raton, Fla. : [S.1.]: CRC Press; IEEE Press, 2000.

[13] N. Baddour and U. Chouinard, "Theory and operational rules for the discrete Hankel transform," JOSA A, vol. 32, no. 4, pp. 611-622, Apr. 2015. 
